\title{
Analysis of Fusarium graminearum Species Complex from Wheat-Maize Rotation Regions in Henan (China)
}

Jun Jie Hao, Shu Na Xie, Jing Sun, Gong Qiang Yang, Jia Zhong Liu, Fei Xu, Yan Yan Ru, and Yu Li Song, Plant Protection Institute, Henan Academy of Agricultural Sciences/Key Laboratory of Integrated Pest Management on Crops in Southern Region of North China/Henan Key Laboratory of Crop Pest Control, Zhengzhou 450002, China

\begin{abstract}
Fusarium head blight (FHB) and maize stalk rot (MSR), caused by members of the Fusarium graminearum species complex (FGSC), are among the most destructive and economically important diseases in the world. Species identity and the trichothecene chemotype of 312 members of the FGSC from diseased wheat spikes and maize stalks in Henan was determined using phylogenetic analyses and a polymerase chain reaction trichothecene chemotype assay. F. graminearum sensu stricto accounted for more than $93 \%$ of the FGSC isolates associated with FHB $(N=168)$ and MSR $(N=130)$. The remaining isolates were $F$. asiaticum. Significant differences were found in the frequencies of the two species within the hosts $(P<0.01)$. However, the frequencies of the same species in FHB and MSR were similar $(P>$

$0.05)$ for wheat and maize isolates, indicating that the composition of the FGSC with respect to wheat and maize in these fields varied little. The 15acetyl-deoxynivalenol (15-ADON) trichothecene chemotype represented 92.7 and $98.5 \%$ of isolates from wheat $(N=167)$ and maize $(N=130)$, respectively. However, the 3-acetyl-deoxynivalenol chemotype was found in $6.7 \%$ of wheat isolates, and the nivalenol chemotype in $1.5 \%$ of MSR isolates and in $0.6 \%$ of FHB isolates. Mycelial growth at different concentrations of carbendazim and difenoconazole did not differ between $F$. graminearum sensu stricto and $F$. asiaticum. These results suggest that the 15-ADON chemotype of $F$. graminearum sensu stricto is the predominant pathogen that causes wheat- and maize-related diseases in this region.
\end{abstract}

Fusarium graminearum species complex (FGSC) is one of several common pathogens that can cause a variety of root, stalk, and ear rot diseases in crops. It not only can lead to a reduction in crop yield but also can produce a variety of mycotoxins that can enter the food chain. The FGSC contains at least 16 distinct species. Some species have specific geographical distributions. $F$. asiaticum and $F$. graminearum sensu stricto are abundant in China (O'Donnell et al. 2000, 2004; Sarver et al. 2011; Yli-Mattila et al. 2009; Zhang et al. 2012). Studies on species of the FGSC causing Fusarium head blight (FHB) in China have shown that $F$. asiaticum is dominant in the Yangtze River Basin and in the southwest of China, whereas $F$. graminearum sensu stricto is predominant in northeast China and in the Huanghuaihai region of northern and central China (Zhang et al. 2012).

Related studies have found that members of the FGSC exhibit host preference on wheat, maize, barley, and rice in certain regions. In China, Korea, and Brazil, F. asiaticum was in the majority of FGSC on wheat or rice in southern regions (Gomes et al. 2015; Lee et al. 2009; Zhang et al. 2012), F. meridionale was the predominant species on ears and stalks of maize in Brazil (Kuhnem et al. 2016), and $F$. graminearum sensu stricto was the predominant species on maize in Korea (Lee et al. 2012) and on wheat and maize roots in South Africa (Boutigny et al. 2011). However, F. graminearum sensu stricto is dominant on wheat in northern China and Brazil (Del Ponte et al. 2015; Zhang et al. 2012). The pathogen diversity of the FGSC appears to be host, region, agroecosystem, and climate dependent; however, the real reasons are not clear.

Trichothecenes produced by the FGSC not only reduce the safety of food and feed but also are related to the virulence of each pathogen. The FGSC mainly produces type B trichothecene mycotoxins, including nivalenol (NIV), 3-acetyl-deoxynivalenol (3-ADON), and 15-acetyl-deoxynivalenol (15-ADON) (Goswami and Kistler 2005; Miller et al. 1991; Ward et al. 2002). In China, studies related to the FGSC causing FHB indicated that $F$. graminearum sensu

Corresponding authors: J. J. Hao; E-mail: haojjds@163.com; Y. L. Song; E-mail: songyuli2000@126.com

Accepted for publication 6 December 2016.

Copyright $(\odot 2017$ The Author(s). This is an open access article distributed under the CC BY-NC-ND 4.0 International license. stricto mainly produces $15-\mathrm{ADON}$ and that $F$. asiaticum mainly produces 3-ADON or NIV (Zhang et al. 2007; Zhang et al. 2012; Zhang et al. 2013).

Henan, in northern and central China, was chosen because it is the largest winter wheat-summer maize rotation area $\left(5.3\right.$ million $\mathrm{hm}^{2}$ of wheat and 3.3 million $\mathrm{hm}^{2}$ of maize) in the Huanghuaihai region. The wheat-maize cropping area covers more than 3.0 million $\mathrm{hm}^{2}$. Crop rotation, conservation tillage, and crop residue management may have contributed to the increase of FHB and maize stalk rot (MSR) in this region.

Studies have been carried out on FHB to assess population structure, trichothecene chemotype, and geographical distributions of the FGSC. However, information is limited on the FGSC from maize fields in China. Consequently, in the present study, we aim to determine the differences in the FGSC composition, including the chemotypes of each species and their geographical distribution between FHB and MSR in wheat-maize cropping areas of Henan, and to assess whether $F$. asiaticum and $F$. graminearum sensu stricto differ in their pathogenicity and fungicide sensitivity toward wheat and maize.

\section{Materials and Methods}

Isolate collection. Wheat spikes and maize basal stalks that exhibited symptoms typical of FHB and MSR were harvested from wheat-maize rotation fields spanning 50 counties in Henan during 2011, 2012, and 2013 (Fig. 1).

Isolation, culture, and single-sporing followed the protocol described for MSR and FHB in Sun et al. (2014) and Gale et al. (2002), respectively. Fusarium isolates were initially identified morphologically (Leslie and Summerell 2006) and validated by speciesspecific polymerase chain reaction (PCR) assay (Rahjoo et al. 2008; Sun et al. 2014). DNA sequence-based identification of unidentified isolates was achieved using the translation elongation factor $1 \alpha(E F$ $1 \alpha$ ) gene. DNA sequences were edited in SeqMan II 4.03 (Lasergene Sequence Analysis Software; DNASTAR), then searched for similarities using the BLAST similarity search programs in the National Center for Biotechnology Information and the FUSARIUM-ID v.1.0 database (http://fusarium.cbio.psu.edu) (Geiser et al. 2004). Isolates were purified using single sporing and stored for use. This procedure identified 312 isolates as members of the FGSC.

DNA extraction. Each isolate was grown on a potato dextrose agar (PDA) plate at $25^{\circ} \mathrm{C}$ for 1 week. The mycelium from each plate 
was harvested, frozen in liquid nitrogen, and then ground into fine powder. Genomic DNA from each isolate was extracted using a commercial fungal extraction kit (Shanghai Lifefeng Biotech) and stored at $-20^{\circ} \mathrm{C}$.

PCR amplification and sequencing. PCR amplification and sequencing of the $E F-1 \alpha$ gene and the 3-O-acetyltransferase (Tri101) gene were conducted using the primer pair EF1/EF2 (O'Donnell et al. 1998) and TRI1015B/TRI1013E (Proctor et al. 2009), respectively (Table 1). The sequencing method of O'Donnell et al. (2004) was followed and the PCR products were sequenced by Shanghai Sangon Biological Engineering Co., Ltd.

Phylogenetic analysis. DNA sequences were aligned and adjusted manually using DNAStar-SeqMan software. Reference sequences of the EF-1 $\alpha$ gene and TrilO1 gene representing all known NRRL strains of 16 clade species of the FGSC were downloaded from the GenBank database. Sequences were aligned in Clustal X 1.81 (www.clustal.org/ clustal2/). Phylogenetic relationships were inferred in MEGA6.06 (Tamura et al. 2013) using the maximum-likelihood method with 1,000 bootstrap replicates. The best-fit model of molecular evolution was selected based on estimation of Bayesian Information Criterion scores.

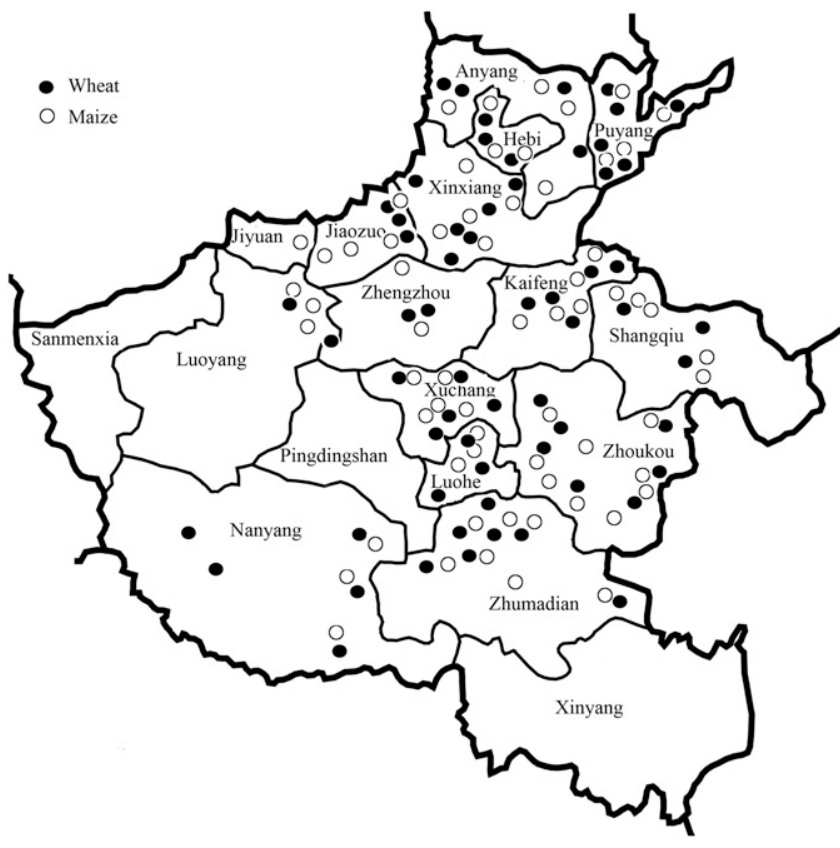

Fig. 1. Map showing field samples of Fusarium head blight and maize stalk rot from the wheat-maize rotation region of Henan in 2011, 2012, and 2013.
Determination of trichothecene chemotypes. Trichothecene chemotypes were generated by multiplex PCR targeting Tri3 and Tri12 gene sequences under the conditions described by Starkey et al. (2007). PCR delineated FGSC and the other B-trichothecene producers as either the 3-ADON, 15-ADON, or NIV trichothecene chemotype. The Tri3 gene primers were CON, 3NA, 3D15A, and 3D3A (Table 1). These primers produced amplified target DNA of approximately 840,610 , and 243 bp that contained the NIV, 15ADON, and 3-ADON chemotype, respectively. The Tri12 primers used were $12 \mathrm{CON}, 12 \mathrm{NF}, 12-15 \mathrm{~F}$, and $12-3 \mathrm{~F}$. These primers generated amplified target DNA of approximately 840, 670, and $410 \mathrm{bp}$ that contained the NIV, 15-ADON, and 3-ADON chemotype, respectively (Table 1).

Pathogenicity assays. In all, 10 isolates of $F$. graminearum sensu stricto and $F$. asiaticum were analyzed for their pathogenicity on corn and wheat using the Petri plate assay of Broders et al. (2007) and Hao et al. (2013). Isolates were grown on PDA at $25^{\circ} \mathrm{C}$ for 4 days. A colony ( $5 \mathrm{~mm}$ in diameter) from each isolate was transferred to the center of a Petri dish ( $8 \mathrm{~mm}$ in diameter) which contained $15 \mathrm{ml}$ of $1.7 \%$ water agar. The isolates were grown for 4 days. In total, 10 seeds of either corn hybrid 'Xundan26' or 20 seeds of wheat 'Zhengmai366' were surface disinfested in $3 \% \mathrm{NaClO}$ and then plates were filled to within $1 \mathrm{~cm}$ of the rim. The plates were incubated at $25^{\circ} \mathrm{C}$ in darkness for 7 days. Percent seed germination and rot was determined according to the following scale: 1 = seed germinated, healthy seedlings were produced with no clear symptoms of infection; $2=$ seed germination observed, rarely with signs of infection, 1 to $19 \%$ with lesions on seedling root; 3 = seed germinated, partially covered with mycelium, 20 to $75 \%$ with lesions on seedling root; $4=$ seed germinated, completely covered with mycelium, more than $75 \%$ with lesions on seedling root; and $5=$ seed did not germinate, completely covered with mycelium. We used three block replicates for each treatment (10 isolates and one control). There were three replicate plates for each isolate in each experiment. The entire procedure was repeated three times.

Fungicide sensitivity. The same 10 isolates used in the pathogenicity assay were used to assess sensitivity to carbendazim and difenoconazole (Shenyang Academy of Chemistry and Industry). Carbendazim and difenoconazole were dissolved in $\mathrm{HCl}$ and acetone, respectively. This stock solution was added to PDA cooled to approximately $55^{\circ} \mathrm{C}$. Technical-grade carbendazim was evaluated at $0.1,0.15$, and $0.25 \mu \mathrm{g} / \mathrm{ml}$, and unamended PDA was used as the control. Difenoconazole (technical-grade product) was evaluated at $0.5,2.5$, and $10 \mu \mathrm{g} / \mathrm{ml}$, and unamended PDA was again used as the control. A colonized plug ( $5 \mathrm{~mm}$ in diameter) taken from the edge of each 6-day-old colony was transferred to the center of a plate containing each concentration of the fungicide. The colony diameter was measured in two places after 6 days of incubation at $25^{\circ} \mathrm{C}$. For each isolate at each concentration, the percent growth inhibition was calculated by dividing the average colony diameter minus $5 \mathrm{~mm}$ for the

Table 1. Polymerase chain reaction primers used in the experiments

\begin{tabular}{|c|c|c|c|c|}
\hline Primer name & Primer sequence $\left(5^{\prime}-3^{\prime}\right)^{x}$ & Amplified target DNA (bp) ${ }^{\mathbf{y}}$ & Target gene & Citation $^{\mathrm{z}}$ \\
\hline$\overline{\mathrm{EF} 1}$ & ATGGGTAAGGARGACAAGAC & 600 & $E F-1 \alpha$ & O'Donnell et al. 1998 \\
\hline $\mathrm{EF} 2$ & GGARGTACCAGTSATCAGTT & $\ldots$ & $\ldots$ & $\ldots$ \\
\hline TRI1015B & CCATGGGTCGCRGGCCARGTSAA & 1,336 & TRIIO1 & Proctor et al. 2009 \\
\hline TRI1013E & AACTCSCCRTCIGGYTTYTTNGGCAT & $\ldots$ & $\ldots$ & $\ldots$ \\
\hline $3 \mathrm{CON}$ & TGGCAAAGACTGGTTCAC & 840 (NIV) & Tri3 & Starkey et al. 2007 \\
\hline $3 \mathrm{NA}$ & GTGCACAGAATATACGAGC & $610(15-\mathrm{ADON})$ & $\ldots$ & $\ldots$ \\
\hline $3 \mathrm{D} 15 \mathrm{~A}$ & ACTGACCCAAGCTGCCATC & $243(3-\mathrm{ADON})$ & $\ldots$ & $\ldots$ \\
\hline 3D3A & CGCATTGGCTAACACATG & $\ldots$ & $\ldots$ & $\ldots$ \\
\hline $12 \mathrm{CON}$ & CATGAGCATGGTGATGTC & 840 (NIV) & Tri12 & Starkey et al. 2007 \\
\hline $12 \mathrm{NF}$ & TCTCCTCGTTGTATCTGG & $670(15-\mathrm{ADON})$ & $\ldots$ & $\ldots$ \\
\hline $12-15 \mathrm{~F}$ & TACAGCGGTCGCAACTTC & $410(3-\mathrm{ADON})$ & $\ldots$ & $\ldots$ \\
\hline $12-3 \mathrm{~F}$ & CTTTGGCAAGCCCGTGCA & $\ldots$ & $\ldots$ & $\ldots$ \\
\hline
\end{tabular}

${ }^{\mathrm{x}} \mathrm{R}=\mathrm{A}+\mathrm{G}$ and $\mathrm{Y}=\mathrm{C}+\mathrm{T}$.

y Nivalenol (NIV), 3-acetyl-deoxynivalenol (3-ADON), and 15-acetyl-deoxynivalenol (15-ADON) chemotypes.

${ }^{\mathrm{z}}$ Reference from literature cited. 
agar plug by the average colony diameter on nonfungicide amended media. The results were multiplied by 100 . Three replicates were performed for each of the selected isolates. Tests were repeated twice. The results were analyzed using PROC GLM of SAS (v 9.2; SAS Institute, Inc.). These included analyzing the variation in sensitivity of isolates and species to the two different fungicides and the effect of each fungicide concentration on the efficacy of the two fungicides.

\section{Results}

Species diversity. According to morphological and molecular identification, 494 Fusarium isolates were collected by sampling wheat-maize rotation fields in Henan during the 2011, 2012, and 2013 growing seasons. In all, 180 isolates of the FGSC from FHB samples were collected and identified. Among the 314 isolates from MSR samples, the FGSC was most abundant $(42.0 \%, N=132)$ of isolates, followed by $F$. proliferatum $(29.9 \%, N=94)$ and $F$. verticillioides $(28.0 \%, N=88)$. Among the 312 isolates of the FGSC collected in this study, 180 isolates from wheat and 132 isolates from maize were nested within the FGSC.

Phylogenetic analysis of $E F-1 \alpha$ and Tril01 gene sequences revealed that $F$. graminearum sensu stricto and $F$. asiaticum were recovered in our study of FHB and MSR in wheat-maize rotation fields in Henan. Among 312 isolates, 14 isolates (4.5\%) belonged to $F$. asiaticum. The remaining $298(95.5 \%)$ isolates belonged to $F$. graminearum sensu stricto (Fig. 2; Table 2). Among the isolates from wheat $(N=180)$, the majority were $F$. graminearum sensu

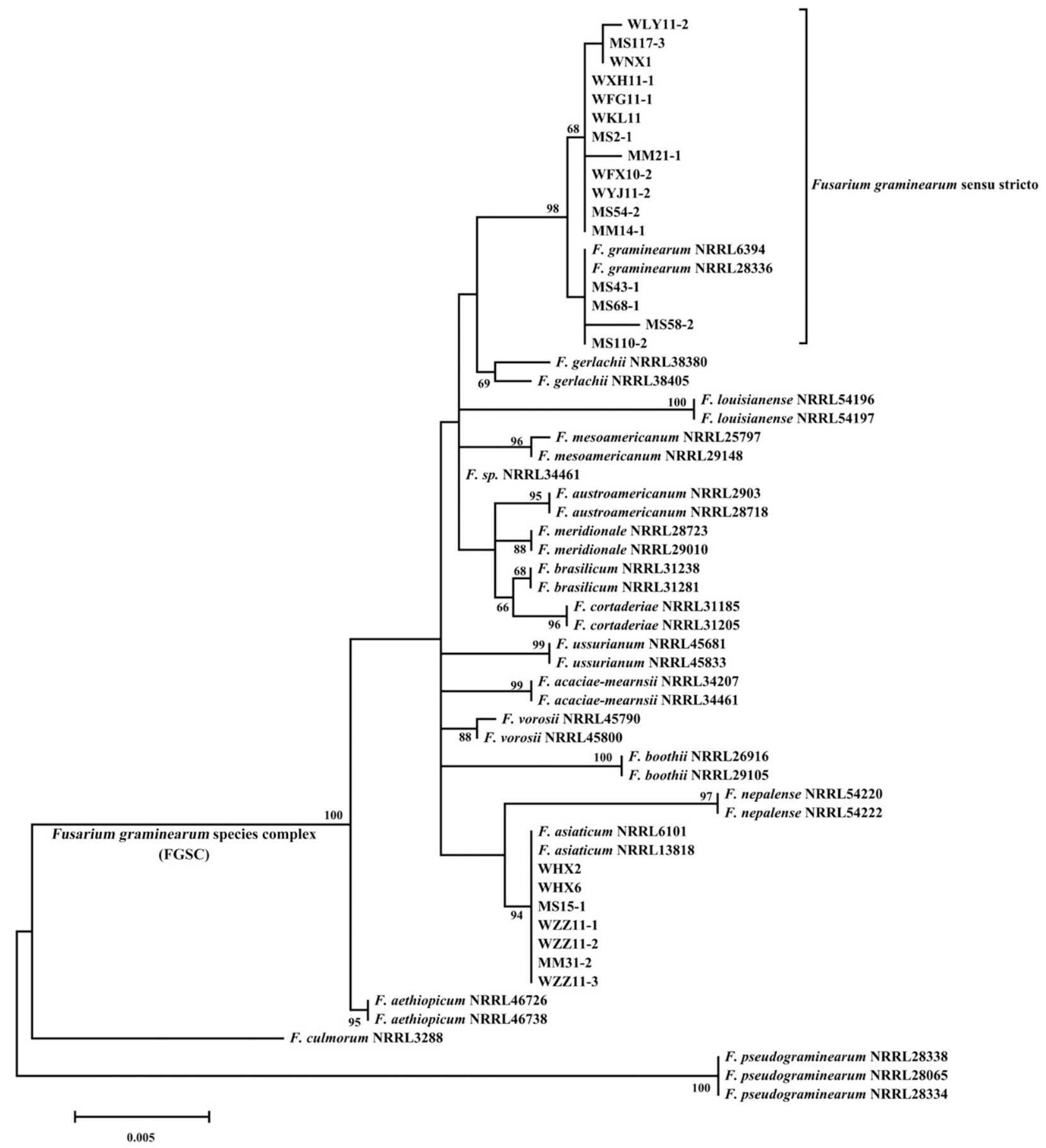

Fig. 2. Phylogeny of Fusarium graminearum species complex based on the translation elongation factor $1 \alpha(E F-1 \alpha)$ gene and the 3-0-acetyltransferase (Tri101) gene region. Bootstrap values (percentage, based on 1,000 replications) $\geq 60$ are shown on branches. Some representative strains of $F$. graminearum species complex isolates in this study were selected in the phylogenetic tree with "W" and "M" codes obtained from wheat and maize, respectively. The remaining isolates were from the National Center for Biotechnology Information. F. pseudograminearum and one F. culmorum were used as outgroups. 
stricto $(93.3 \%, N=168)$ but $6.7 \%$ were $F$. asiaticum $(N=12)$. Among 132 isolates from MSR, only 2 isolates (1.5\%), from Luoyang City and Zhumadian City, were $F$. asiaticum. The remaining isolates $(98.5 \%, N=130)$ were $F$. graminearum sensu stricto (Table 2$)$. Significant differences were found in the frequencies of $F$. asiaticum and $F$. graminearum sensu stricto by host $(P<0.01)$. However, the frequency of these species causing FHB and MSR were similar $(P>$ $0.05)$ to those shown for wheat and maize isolates. This shows that the FGSC composition is similar for wheat and maize in the wheat-maize rotation fields in Henan. $F$. graminearum sensu stricto was the predominant FHB and MSR pathogen in Henan.

Trichothecene chemotypes. The trichothecene chemotype of 312 isolates was determined using two multiple PCR targeting Tri3 and Tri12. The 15-ADON chemotype was predominant in isolates from wheat $(92.7 \%)$ and maize $(98.5 \%)$ in Henan and accounted for 297 $(95.1 \%)$ of isolates (Table 3$)$. The NIV chemotype comprised $0.6 \%(N=1)$ of isolates from wheat and $1.5 \%(N=2)$ of isolates from maize. In total, 12 isolates $(6.7 \%)$ from wheat were the 3 -ADON chemotype. The frequency of the 15 -ADON or NIV chemotype among isolates from wheat and maize was not significantly different $(P>0.05)$. The 15-ADON chemotype was observed throughout Henan and was the most common chemotype in the wheat-maize fields.

Results indicated that, among the 168 isolates of $F$. graminearum sensu stricto from wheat, 167 isolates possessed the 15-ADON and 1 the NIV chemotype. All 12 isolates of $F$. asiaticum were the 3ADON chemotype. In addition, 130 isolates of $F$. graminearum sensu stricto from maize were 15-ADON chemotype and 2 isolates of $F$. asiaticum were the NIV chemotype (Table 4).

Pathogenicity. All 10 representative isolates of the FGSC exhibited a high degree of pathogenicity to both maize and wheat when grown on Petri plates under the described conditions. Pathogenicity was scored 4, seed had germinated and were completely covered with mycelium, and more than $75 \%$ contained lesions on the seedling roots. $F$. graminearum sensu stricto and $F$. asiaticum also did not differ significantly in degree of pathogenicity on both corn and wheat when using the Petri plate assay (all data not shown).

Fungicide sensitivity. Analysis of variance showed that there was no difference between $F$. graminearum sensu stricto and $F$. asiaticum species in inhibiting mycelial growth at different concentrations of carbendazim or difenoconazole (Table 5). No significant difference was observed among the three $F$. asiaticum isolates $(P>0.05)$ but there was a significant difference $(P<0.01)$ among the seven $F$. graminearum sensu stricto isolates. Highly significant differences $(P<$ $0.01)$ were observed between fungicides and between their different

Table 2. Diversity of Fusarium graminearum species complex within hosts in wheat-maize rotation region of Henan ${ }^{\mathrm{z}}$

\begin{tabular}{lccc}
\hline & \multicolumn{2}{c}{ Isolates (\%) } \\
\cline { 2 - 3 } Host & $\begin{array}{c}\text { F. graminearum } \\
\text { sensu stricto }\end{array}$ & $\boldsymbol{F}$. asiaticum & $\boldsymbol{N}$ \\
\hline Wheat & 93.3 & 6.7 & 180 \\
Maize & 98.5 & 1.5 & 132 \\
\hline
\end{tabular}

${ }^{\mathrm{z}}$ Results are expressed as percentage of isolates relative to the total number of isolates $(N)$.

Table 3. Chemotype of Fusarium graminearum species complex isolates collected from wheat and maize in wheat-maize rotation region of Henan ${ }^{y}$

\begin{tabular}{lcccc}
\hline & \multicolumn{3}{c}{ Isolates (\%) per chemotype $^{\mathbf{z}}$} & \\
\cline { 2 - 4 } Host & 15-ADON & 3-ADON & NIV & $\boldsymbol{N}$ \\
\hline Wheat & 92.7 & 6.7 & 0.6 & 180 \\
Maize & 98.5 & 0.0 & 1.5 & 132 \\
\hline
\end{tabular}

${ }^{\mathrm{y}}$ Results are expressed as percentage of isolates relative to the total number of isolates $(N)$.

${ }^{z}$ Trichothecene chemotypes: 3-acetyl-deoxynivalenol (3-ADON), 15-acetyldeoxynivalenol (15-ADON), and nivalenol (NIV). concentrations. The effect of fungicide-concentration was also highly significant (Table 5).

There was a significant difference in the inhibition of mycelial growth at different carbendazim and difenoconazole concentrations (Table 6). An increase in concentration led to a significant increase in inhibition efficacy. Difenoconazole inhibited mycelial growth to $91.2 \%$ of the control at a concentration of $10 \mu \mathrm{g} / \mathrm{ml}$. However, mycelial growth of 35 to $83.6 \%$ of the control was observed on media supplemented with carbendazim at a concentration of either 0.1 , 0.15 , or $0.25 \mu \mathrm{g} / \mathrm{ml}$ (Table 6). Additionally, the two fungicides provided greater inhibition in WLY11-2-1, MSP11-2-1, and MS105-6 isolates than MS29-1, WZX3, WPY11-3-1, and MS68-1 within the seven isolates of $F$. graminearum sensu stricto (Table 7).

\section{Discussion}

This study has described pathogen diversity of the FGSC in wheatmaize rotation fields in Henan for the first time and increased the body of information on pathogen detection and diversity of the FGSC. Phylogenetic analysis using the $E F-1 \alpha$ gene and Tril01 gene region sequences revealed two species within the FGSC, $F$. graminearum sensu stricto and $F$. asiaticum, from a winter wheat-summer maize cropping area in Huanghuaihai region. This is the first report of $F$. graminearum sensu stricto and $F$. asiaticum causing MSR in the world. Isolates used in this study were mainly collected from a winter

Table 4. Chemotypes of Fusarium graminearum sensu stricto ( $F$. graminearum) and $F$. asiaticum from wheat and maize in wheat-maize rotation region of Henany

\begin{tabular}{lccccc}
\hline & \multicolumn{2}{c}{ Wheat } & & \multicolumn{2}{c}{ Maize } \\
\cline { 2 - 3 } \cline { 5 - 6 } Chemotypes $^{\mathbf{z}}$ & $\boldsymbol{F}$. graminearum & $\boldsymbol{F}$. asiaticum & & $\boldsymbol{F}$. graminearum & $\boldsymbol{F}$. asiaticum \\
\hline $3-A D O N$ & 0 & 12 & & 0 & 0 \\
$15-A D O N$ & 167 & 0 & & 130 & 0 \\
NIV & 1 & 0 & & 0 & 2 \\
$N$ & 168 & 12 & & 130 & 2
\end{tabular}

${ }^{y}$ Results are expressed as number of isolates relative to the total number of isolates $(N)$.

${ }^{\mathrm{z}}$ Trichothecene chemotypes: 3-acetyl-deoxynivalenol (3-ADON), 15-acetyldeoxynivalenol (15-ADON), and nivalenol (NIV).

Table 5. Analysis of variance for Fusarium asiaticum and F. graminearum sensu stricto on fungicide sensitivity

\begin{tabular}{lrrrc}
\hline Source & df & \multicolumn{1}{c}{ MS } & $\boldsymbol{F}$ & $\boldsymbol{P}$ value \\
\hline Rep & 2 & 38.9 & 0.8 & 0.459 \\
Between species & 1 & 42.4 & 0.9 & 0.357 \\
Isolates (species) & 8 & 239.9 & 4.8 & $<0.0001$ \\
$\quad$ Within F. asiaticum & 2 & 35.7 & 0.7 & 0.489 \\
$\quad$ Within F. graminearum & 6 & 308.0 & 6.2 & $<0.0001$ \\
$\quad$ sensu stricto & & & & \\
Fungicide & 1 & $2,883.4$ & 58.0 & $<0.0001$ \\
Concentration & 2 & $44,348.2$ & 892.1 & $<0.0001$ \\
Fungicide $\times$ concentration & 2 & $1,966.3$ & 39.6 & $<0.0001$ \\
Error & 163 & 49.7 & $\ldots$ & $\ldots$ \\
\hline
\end{tabular}

Table 6. Growth of Fusarium asiaticum and $F$. graminearum sensu stricto evaluated on media amended with different concentrations of difenoconazole and carbendazim ${ }^{\mathrm{z}}$

\begin{tabular}{lcc}
\hline Fungicide & $\begin{array}{c}\text { Concentration } \\
(\boldsymbol{\mu} \mathbf{g} / \mathbf{m} \mathbf{l})\end{array}$ & $\begin{array}{c}\text { Inhibition of mycelial } \\
\text { growth to the control }(\boldsymbol{\%})\end{array}$ \\
\hline Difenoconazole & 0.5 & $31.4 \mathrm{c}$ \\
& 2.5 & $68.0 \mathrm{~b}$ \\
Carbendazim & 10.0 & $91.2 \mathrm{a}$ \\
& 0.1 & $35.0 \mathrm{c}$ \\
& 0.15 & $48.3 \mathrm{~b}$ \\
& 0.25 & $83.6 \mathrm{a}$
\end{tabular}

${ }^{\mathrm{z}}$ Means in a column followed by the same letter do not different significantly $(P=0.05)$. 
wheat-summer maize cropping area under a double cropping system. Previous studies focused on Chinese wheat scab but little research has been conducted on maize FGSC in the wheat-maize cropping system, especially as it relates to a comparative study of FGSC between wheat and maize. Previous studies associated with wheat scab have shown that $F$. graminearum sensu stricto is the predominant species in the north of China, whereas $F$. asiaticum is predominant in the south. The cooler temperatures (annual average temperature $15^{\circ} \mathrm{C}$ or lower) in the north of China appear to favor $F$. graminearum sensu stricto and the warmer south (annual average temperature above $15^{\circ} \mathrm{C}$ ) appears to favor $F$. asiaticum. The majority of $F$. asiaticum isolates were collected from these warmer regions in which FHB epidemics occurred often (Gale et al. 2002; Zhang et al. 2007; Zhang et al. 2012; Zhang et al. 2013). All isolates from the Zhejiang wheat fields in eastern China were $F$. asiaticum (Gale et al. 2002). Analysis of wheat scab FGSC from 15 provinces in China found that $F$. graminearum sensu stricto and $F$. asiaticum coexist in most provinces of the Yangtze River Basin and the Huaihe River Basin (Zhang et al. 2012). However, F. asiaticum was dominant in the Yangtze River Basin and in southwestern China. $F$. graminearum sensu stricto was dominant in the northeast and Huanghuaihai regions. An obvious shift in species domination from $F$. asiaticum to $F$. graminearum sensu stricto from south to north existed; $35 F$. graminearum sensu stricto and $3 F$. asiaticum isolates were identified from 20 sampling points in the Henan wheat area (Zhang et al. 2012). These results are consistent with this study, which demonstrates the presence of $F$. graminearum sensu stricto and $F$. asiaticum in wheat scab in Henan. $F$. graminearum sensu stricto was the dominant pathogen. The distribution of $F$. asiaticum did not have a defined geographical distribution, which is likely due to the limited number of sampling points.

Ndoye et al. (2012) studied maize ear rot in China and showed that the NIV chemotype of $F$. asiaticum was dominant in the warmer south and 15-ADON chemotype of $F$. graminearum sensu stricto was dominant in the colder north. However, the toxin chemotype of $F$. asiaticum from wheat in the same region was different. We have found that, for both wheat and maize, the dominant species was 15ADON chemotype of $F$. graminearum sensu stricto. The FGSC from maize ear rot in South Korea contained F. meridionale, F. boothii, $F$. asiaticum, and $F$. graminearum sensu stricto (Lee et al. 2012); $F$. graminearum sensu stricto was the dominant species $(75 \%)$ but in Korean rice, F. asiaticum was the dominant species (78.5\%), followed by $F$. graminearum sensu stricto (Lee et al. 2009). There are significant differences between hosts; the authors believed that F. asiaticum had a preference and adapted to rice (Lee et al. 2012). Boutigny et al. (2011), studying species-specific differences in host preference, found that, in South Africa, $F$. graminearum sensu stricto, $F$. boothii, and $F$. meridionale were the three dominant

Table 7. Effect of Fusarium asiaticum and $F$. graminearum sensu stricto on fungicide sensitivity

\begin{tabular}{|c|c|c|c|}
\hline Species, isolate & Host & Chemotype $^{\mathrm{y}}$ & $\begin{array}{l}\text { Inhibition of } \\
\text { growth }(\%)^{\mathrm{z}}\end{array}$ \\
\hline \multicolumn{4}{|l|}{ F. asiaticum } \\
\hline WZZ11-2-1 & Wheat & 3-ADON & $59.9 \mathrm{a}$ \\
\hline WZX1 & Wheat & 3-ADON & $61.8 \mathrm{a}$ \\
\hline WHX2-5 & Wheat & 3-ADON & $59.1 \mathrm{a}$ \\
\hline \multicolumn{4}{|c|}{ F. graminearum sensu stricto } \\
\hline MS105-6 & Maize & $15-\mathrm{ADON}$ & $62.9 \mathrm{a}$ \\
\hline MS29-1 & Maize & $15-\mathrm{ADON}$ & $58.2 \mathrm{~b}$ \\
\hline MSP11-2-1 & Wheat & $15-\mathrm{ADON}$ & $63.1 \mathrm{a}$ \\
\hline WLY11-2-1 & Wheat & $15-\mathrm{ADON}$ & $64.1 \mathrm{a}$ \\
\hline WZX3 & Wheat & $15-A D O N$ & $56.4 \mathrm{bc}$ \\
\hline WPY11-3-1 & Wheat & $15-\mathrm{ADON}$ & $53.6 \mathrm{bc}$ \\
\hline MS68-1 & Maize & $15-\mathrm{ADON}$ & $56.1 \mathrm{bc}$ \\
\hline
\end{tabular}

${ }^{\mathrm{y}}$ Trichothecene chemotypes: 3-acetyl-deoxynivalenol (3-ADON) and 15acetyl-deoxynivalenol (15-ADON).

${ }^{\mathrm{z}}$ Inhibition of mycelial growth to the control. Means followed by the same letter do not different significantly $(P=0.05)$. species in the FGSC from wheat, maize, and barley. F. graminearum sensu stricto was the dominant species in wheat and barley. F. boothii was the dominant species in maize ears. $F$. graminearum sensu stricto was the dominant species in maize roots, followed by $F$. boothii and F. meridionale, showing that different species in the same area of South Africa had certain host preferences and adaptability. In Brazil, $F$. graminearum sensu stricto is dominant in wheat (Astolfi et al. 2012; Del Ponte et al. 2015), F. asiaticum is dominant in rice (Gomes et al. 2015), and F. meridionale is the predominant species on maize ears and stalks (Kuhnem et al. 2016).

Among the 132 isolates obtained from MSR samples, this study has identified only two NIV-type $F$. asiaticum isolates from Zhumadian and Luoyang. In addition, 12 3-ADON-type $F$. asiaticum isolates were collected from Zhengzhou, Hebi, and Zhumadian. One NIVtype isolate of $F$. graminearum sensu stricto was found in wheat in Hebi, while the other $F$. graminearum sensu stricto isolates produced $15-$ ADON chemotypes. Studies have shown that $F$. graminearum sensu stricto populations from Asia mainly produced 15-ADON, $F$. asiaticum populations from Asia mainly produced 3-ADON or NIV chemotypes, and NIV-type $F$. graminearum sensu stricto populations have not be found (Gale et al. 2002; Lee et al. 2009, 2012; Shen et al. 2012; Zhang et al. 2007; Zhang et al. 2012; Zhang et al. 2013). Gale et al. (2011) reported NIV-type $F$. graminearum sensu stricto populations where $79 \%$ of the isolates were prevalent on wheat in Louisiana. Our study is the first to demonstrate the existence of the NIV chemotype of $F$. graminearum sensu stricto in China.

From maize and wheat, although we obtained 2 and $12 F$. asiaticum isolates and 130 and $168 \mathrm{~F}$. graminearum sensu stricto isolates, respectively, no significant difference in frequency between isolates of $F$. asiaticum and $F$. graminearum sensu stricto between wheat and maize was found. A significant difference was observed in the frequency between the $F$. asiaticum and $F$. graminearum sensu stricto isolates within the same host. However, from species and chemotype studies, we found a difference in host between 3-ADON-type $F$. asiaticum strains. This may be the result of a limited number of sampling points and can be addressed by expanding the geographical range of the sampling points and the sample size. This study shows that the 15-ADON-type $F$. graminearum sensu stricto is the main pathogen that causes wheat- and maize-related diseases in Henan. Additional research on the effect of plant residue and tilling method on these related diseases will be of economic significance, because the FGSC can cause root, stalk, and ear rot diseases in wheat-maize rotation cropping fields.

This study identified isolates from wheat and maize and assayed their pathogenicity on both maize and wheat using a Petri plate assay; this method was also used to analyze differences in pathogenicity between different isolates of Fusarium on maize and soybeans (Broders et al. 2007; Hao et al. 2013). The results of this study have shown that the representative isolates all were scored 4; no difference between species was observed between chemotypes and between the isolates studied. Lamprecht et al. (2011) found a difference in pathogenicity between $F$. boothii, $F$. graminearum sensu stricto, and $F$. meridionale in causing maize root rot. In addition, these representative isolates did not show significant differences in inhibition of mycelial growth at the different concentrations of carbendazim and difenoconazole between species or between chemotypes, which was similar to the results of Tateishi et al. (2010), who analyzed metconazole sensitivity of $F$. asiaticum and $F$. graminearum sensu stricto from wheat in Japan. However, Zhang et al. (2013), who studied wheat scab FGSC from Jiangsu Province, China, found that the majority of NIV and 15-ADON-type isolates were sensitive to carbendazim, whereas many 3-ADON-type isolates were resistant to carbendazim. In addition, Zhang et al. (2012) found that 3-ADON-type isolates of $F$. asiaticum had a greater pathogenicity, growth rate, sporulation capacity, and resistance to benzimidazole than the NIV type of $F$. asiaticum. Isolates selected in the present study were sensitive to both fungicides. This may be a result of the small sample size of representative isolates. We found no isolates that were resistant to carbendazim and difenoconazole. 


\section{Acknowledgments}

This study was supported by the Science-Technology Foundation for Outstanding Young Scientists (2013YQ18) and Special Funds for Scientific Research and Development of Henan Academy of Agricultural Sciences (201412008 and 2016ZC36).

\section{Literature Cited}

Astolfi, P., Reynoso, M. M., Ramirez, M. L., Chulze, S. N., Alves, T. C. A., Tessmann, D. J., and Del Ponte, E. M. 2012. Genetic population structure and trichothecene genotypes of Fusarium graminearum isolated from wheat in southern Brazil. Plant Pathol. 61:289-295.

Boutigny, A. -L., Ward, T. J., Van Coller, G. J., Flett, B., Lamprecht, S. C., O'Donnell, K., and Viljoen, A. 2011. Analysis of the Fusarium graminearum species complex from wheat, barley, and maize in South Africa provides evidence of species-specific differences in host preference. Fungal Genet. Biol. 48:914-920.

Broders, K. D., Lipps, P. E., Paul, P. A., and Dorrance, A. E. 2007. Evaluation of Fusarium graminearum associated with corn and soybean seed and seedling disease in Ohio. Plant Dis. 91:1155-1160.

Del Ponte, E. M., Spolti, P., Ward, T. J., Gomes, L. B., Nicolli, C. P., Kuhnem, P. R., Silva, C. N., and Tessmann, D. J. 2015. Regional and field-specific factors affect the composition of Fusarium head blight pathogens in subtropical no-till wheat agroecosystem of Brazil. Phytopathology 105:246-254.

Gale, L. R., Chen, L. F., Hernick, C. A., Takamura, K., and Kistler, H. C. 2002. Population analysis of Fusarium graminearum from wheat fields in eastern China. Phytopathology 92:1315-1322.

Gale, L. R., Harrison, S. A., Ward, T. J., O’Donnell, K., Milus, E. A., Gale, S. W., and Kistler, H. C. 2011. Nivalenol-type populations of Fusarium graminearum and $F$. asiaticum are prevalent on wheat in southern Louisiana. Phytopathology 101:124-134.

Geiser, D. M., Jimenz Gasco, M. M., Kang, S., Mkalowska, I., Veeraraghavan, N., Ward, T. J., Zhang, N., Kuldau, G. A., and O'Donnell, K. 2004. FUSARIUMIDv.1.0: A DNA sequence database for identifying Fusarium. Eur. J. Plant Pathol. 110:473-479.

Gomes, L. B., Ward, T. J., Badiale-Furlong, E., and Del Ponte, E. M. 2015. Species composition, toxigenic potential and pathogenicity of Fusarium graminearum species complex isolates from southern Brazilian rice. Plant Pathol. 64: 980-987.

Goswami, R. S., and Kistler, H. C. 2005. Pathogenicity and in planta mycotoxin accumulation among members of the Fusarium graminearum species complex on wheat and rice. Phytopathology 95:1397-1404.

Hao, J. J., Liu, J. Z., Sun, J., Xie, S. N., Wang, J., and Wang, J. 2013. Effects of fungicide seed treatments on maize seeding infected with Fusarium species. J. Maize Sci. 21:120-126. (In Chinese)

Kuhnem, P. R., Ward, T. J., Silva, C. N., Spolti, P., Ciliato, M. L., Tessmann, D. J., and Del Ponte, E. M. 2016. Composition and toxigenic potential of the Fusarium graminearum species complex from maize ears, stalks and stubble in Brazil. Plant Pathol. 65:1185-1191.

Lamprecht, S. C., Tewoldemedhin, Y. T., Botha, W. J., and Calitz, F. J. 2011. Fusarium graminearum species complex associated with maize crowns and roots in the KwaZulu-Natal province of South Africa. Plant Dis. 95:1153-1158.

Lee, J., Chang, I. Y., Kim, H., Yun, S. H., Leslie, J. F., and Lee, Y. W. 2009. Genetic diversity and fitness of Fusarium graminearum populations from rice in Korea. Appl. Environ. Microbiol. 75:3289-3295.

Lee, J., Kim, H., Jeon, J. J., Kim, H. S., Zeller, K. A., Carter, L. L. A., Leslie, J. F., and Lee, Y. W. 2012. Population structure of and mycotoxin production by Fusarium graminearum from maize in South Korea. Appl. Environ. Microbiol. 78:2161-2167.

Leslie, J. F., and Summerell, B. A., eds. 2006. The Fusarium Laboratory Manual. Blackwell Publishing, Ames, IA. doi:10.1002/9780470278376

Miller, J. D., Greenalgh, R., Wang, Y., and Lu, M. 1991. Trichothecene chemotype of three Fusarium species. Mycologia 83:121-130.

Ndoye, M., Zhang, J. B., Wang, J. H., Gong, A. D., Li, H. P., Qu, B., Li, S. J., and Liao, Y. C. 2012. Nivalenol and 15-acetyldeoxynivalenol chemotypes of
Fusarium graminearum clade species are prevalent on maize throughout China. J. Phytopathol. 160:519-524.

O’Donnell, K., Kistler, H. C., Cigelink, E., and Ploetz, R. C. 1998. Multiple evolutionary origins of the fungus causing Panama disease of banana: Concordant evidence from nuclear and mitochondrial gene genealogies. Proc. Natl. Acad. Sci. USA 95:2044-2049.

O'Donnell, K., Kistler, H. C., Tacke, B. K., and Casper, H. H. 2000. Gene genealogies reveal global phylogeographic structure and reproductive isolation among lineages of Fusarium graminearum, the fungus causing wheat scab. Proc. Natl. Acad. Sci. USA 97:7905-7910.

O’Donnell, K., Ward, T. J., Geiser, D. M., Kistler, H. C., and Aoki, T. 2004. Genealogical concordance between the mating type locus and seven other nuclear genes supports formal recognition of nine phylogenetically distinct species within the Fusarium graminearum clade. Fungal Genet. Biol. 41: 600-623.

Proctor, R. H., McCormick, S. P., Alexander, N. J., and Desjardins, A. E. 2009. Evidence that a secondary metabolic biosynthetic gene cluster has grown by gene relocation during evolution of the filamentous fungus Fusarium. Mol. Microbiol. 74:1128-1142.

Rahjoo, V., Zad, J., Javan-Nikkhah, M., Gohari, A. M., Okhovvat, S. M., Bihamta, M. R., Razzaghian, J., and Klemsdal, S. S. 2008. Morphological and molecular identification of Fusarium isolated from maize ears in Iran. J. Plant Pathol. 90:463-468.

Sarver, B. A. J., Ward, T. J., Gale, L. R., Broz, K., Kistler, H. C., Aoki, T., Nicholson, P., Carter, J., and O'Donnell, K. 2011. Novel Fusarium head blight pathogens from Nepal and Louisiana revealed by multilocus genealogical concordance. Fungal Genet. Biol. 48:1096-1107.

Shen, C. M., Hu, Y. C., Sun, H. Y., Li, W., Guo, J. H., and Chen, H. G. 2012. Geographic distribution of trichothecene chemotypes of the Fusarium graminearum species complex in major winter wheat production areas of China. Plant Dis. 96:1172-1178.

Starkey, D. E., Ward, T. J., Aoki, T., Gale, L. R., Kistler, H. C., Geiser, D. M., Suga, H., Tóth, B., Varga, J., and O'Donnell, K. 2007. Global molecular surveillance reveals novel Fusarium head blight species and trichothecene toxin diversity. Fungal Genet. Biol. 44:1191-1204.

Sun, J., Xie, S. N., Liu, J. Z., Liu, J. B., Hao, J. J., and Deng, S. Z. 2014. Morphological and molecular identification of Fusarium isolated from basal stalks of maize in Henan Province. Acta Phytopathol. Sin. 44:8-16.

Tamura, K., Stecher, G., Peterson, D., Filipski, A., and Kumar, S. 2013. MEGA6: Molecular evolutionary genetics analysis version 6.0. Mol. Biol. Evol. 30: 2725-2729.

Tateishi, H., Miyake, T., Mori, M., Kimura, R., Sakuma, Y., and Saishoji, T. 2010. Sensitivity of Japanese Fusarium graminearum species complex isolates to metconazole. J. Pestic. Sci. 35:419-430.

Ward, T. J., Bielawski, J. P., Kistler, H. C., Sullivan, E. O., and O’Donnell, K. 2002. Ancestral polymorphism and adaptive evolution in the trichothecene mycotoxin gene cluster of phytopathogenic Fusarium. Proc. Natl. Acad. Sci. USA 99:9278-9283.

Yli-Mattila, T., Gagkaeva, T., Ward, T. J., Aoki, T., Kistler, H. C., and O'Donnell, K. 2009. A novel Asian clade within the Fusarium graminearum species complex includes a newly discovered cereal head blight pathogen from the Russian Far East. Mycologia 101:841-852.

Zhang, H., Lee, T. V., Waalwijk, C., Chen, W., Xu, J., Xu, J., Zhang, Y., and Feng, J. 2012. Population analysis of the Fusarium graminearum species complex from wheat in China show a shift to more aggressive isolates. PLoS One 7: e31722.

Zhang, J. B., Li, H. P., Dang, F. J., Qu, B., Xu, Y. B., Zhao, C. S., and Liao, Y.C. 2007. Determination of the trichothecene mycotoxin chemotypes and associated geographical distribution and phylogenetic species of the Fusarium graminearum clade from China. Mycol. Res. 111:967-975.

Zhang, L. G., Jia, X. J., Chen, C. J., and Zhou, M. G. 2013. Characterization of carbendazim sensitivity and trichothecene chemotypes of Fusarium graminearum in Jiangsu Province of China. Physiol. Mol. Plant Pathol. 84:53-60. 